\title{
Electromagnetic effects on plasma blob-filament transport
}

\author{
Wonjae Lee $^{\mathrm{a} *}$, Justin R. Angus ${ }^{\mathrm{b}}$, Maxim V. Umansky ${ }^{\mathrm{c}}$, Sergei I. Krasheninnikov ${ }^{\mathrm{a}, \mathrm{d}}$ \\ ${ }^{a}$ University of California, San Diego, La Jolla, CA, USA \\ ${ }^{b}$ Naval Research Laboratory, Washington, D.C., USA \\ ${ }^{c}$ Lawrence Livermore National Laboratory, Livermore, CA, USA \\ ${ }^{d}$ Nuclear Research National University MEPhI, Moscow, 115409, Russia
}

Both microscopic and macroscopic impacts of the electromagnetic effects on blob dynamics are considered. Linear stability analysis and nonlinear BOUT++ simulations demonstrate that electromagnetic effects in high temperature or high beta plasmas suppress the resistive drift wave turbulence in the blob when resistivity drops below some certain value. In the course of blob's motion in the SOL its temperature is reduced, which leads to enhancement of resistive effects, so the blob can switch from electromagnetic to electrostatic regime, where resistive drift wave turbulence become important. It is found that inhomogeneity of magnetic curvature or plasma pressure along the filament length leads to bending of the high-beta blob filaments. This is caused by the increase of the propagation time of plasma current (Alfvén time) in higher-density plasma. The effects of sheath boundary conditions on the part of the blob away from the boundary are also diminished by the increased Alfvén time.

PACS: 52.25.Fi, 52.35.Kt, 52.35.Ra, 52.40.Kh

PSI-21 Keywords: SOL transport, Turbulence, Fluid simulation, 3D edge plasma

*Corresponding Author Address: 9500 Gilman Drive, La Jolla, CA 92093, United States

*Corresponding Author e-mail: wol023@ucsd.edu

Presenting Author: Wonjae Lee

Presenting Authore-mail:wol023@ucsd.edu 


\section{Introduction}

Plasma blobs (or blob-filaments) are meso-scale turbulent structures usually observed in the scrape-off layer (SOL) of magnetic confinement devices [1]. An individual blob is characterized by its density $\tilde{n}$, which is higher than the density of the ambient plasma, $n_{0}$, $\left(\tilde{n} / n_{0} \tilde{>} 2-3\right)$ and its filamentary shape stretched along the magnetic field line $\delta / L_{\|}<<1$, where $L_{\|}$and $\delta$ are blob characteristic scale lengths along and across the magnetic field lines. Due to $E \times B$ convective motion caused by the effective gravity force, blobs in tokamaks propagate to the outer side of the torus with the speed $V_{b}$ reaching a few percent of the sound speed [2]. Relative contribution to the SOL plasma transport associated with blobs can be of the order of unity, in particular in far SOL region close to the first wall. Thus, physics on blob-filament is an important ingredient of our basic understanding of plasma transport in the SOL region.

A large body of literature devoted to the theory of blobs exists at this moment. Comprehensive reviews on blob transport theory and experimental observations can be found in [1] and [2]. Theoretical findings show that blob dynamics are sensitive to the interplay between cross-field plasma polarization current and the current along the field lines. In particular, for the case where parallel current is sheath limited, a typical evolution of blob's shape (e.g. mushroom-like, finger-like or balanced shape) depend on the relative size (small, large, medium) of the blob with respect to the characteristic scale, $\delta_{*}=\rho_{s}\left(L_{\|}^{2} / \rho_{s} / R\right)^{1 / 5}$ where $\rho_{s}=c_{s} / \Omega_{i}$ is gyro-Bohm radius, $c_{s}=\sqrt{T_{e} / M_{i}}$ is ion sound speed. However, for open-end or periodic boundary condition blob's shape always evolves to mushroom-like shape. In addition, various parallel current closure schemes, accompanying stability regimes, and velocity scalings have been developed to explain macroscopic dynamics of blob advection [1, 2 and references therein]. 
Recently, Angus et al. (2012) [3] studied the impact of plasma instabilities on blob dynamics and concluded that the coherency of the blob can be substantially limited by the onset of resistive drift wave instability excited in the blob. However, only electrostatic limit was considered in [3]. The main purpose of our paper is to study possible impact of electromagnetic effects on blob dynamics. We notice that most studies of blob dynamics have only been carried out within an electrostatic approximation, which is only adequate for low beta plasmas $\tilde{\beta}=\beta\left(M_{i} / m_{e}\right)<<1$. However, for edge-localized-mode (ELM) filaments, or for blobs in future tokamaks such as ITER, the plasma beta can be comparable to or even larger than the electron-ion mass ratio. Such high-beta plasma blob can cause macroscopic bending of magnetic field lines and modification of sheath impact on blob transport if the characteristic Alfvén time $\tau_{A}=L_{\|} / v_{A}$ becomes larger than the characteristic blob cross-field convection time, $\tau_{\perp}=\delta / V_{b}$. In addition to that, relatively large electron temperature in ELM filaments and electromagnetic perturbations have a synergetic effect of suppressing the resistive drift wave instability in the blobs.

The remainder of our paper is organized as follows: The 3D electromagnetic governing equations are presented in Section 2. Based on these equations, in Section 3 we analyze linear stability of blobs to get an insight in the microscopic effects of resistive drift instability. In Section 4 we discuss the electromagnetic effects on blob macroscopic dynamics and present the BOUT ++ simulation results. We summarize our findings in Section 5.

\section{Governing equations and blob parameters}

We consider a SOL plasma in a Cartesian geometry with a magnetic field following $\hat{b}_{0}=\hat{z}$ direction, and the radial and effective poloidal direction corresponds to $\hat{x}$ and $\hat{y}$ 
direction respectively. Within the Boussinesq approximation, assuming cold ions and isothermal electrons, we get the following equations governing blob dynamics $[3,4]$ :

$$
\begin{aligned}
& n \rho_{s}^{2} \frac{d}{d t} \nabla_{\perp}^{2} \varphi=\frac{\nabla_{\|} J_{\|}}{e}-\frac{g}{\Omega_{i}} \frac{\partial n}{\partial y}, \\
& \frac{d n}{d t}=\frac{\nabla_{\|} J_{\|}}{e}-\frac{g}{\Omega_{i}} \frac{\partial n}{\partial y}, \\
& \frac{d A_{j \|}}{d t}=\frac{e}{m_{e}} \nabla_{\|}^{0} \phi-\frac{T_{e}}{n m_{e}} \nabla_{\|} n+\frac{e}{\sigma_{\|} m_{e}} J_{\|},
\end{aligned}
$$

where the derivative operations are defined as $d / d t=\partial / \partial t+c_{s} \rho_{s} \hat{b}_{0} \times \nabla \varphi \cdot \nabla$ and $\nabla_{\|}=\nabla_{\|}^{0}+\left(\nabla A_{\|} / B_{0}\right) \times \hat{b}_{0} \cdot \nabla, \varphi=e \phi / T_{e}$ is a normalized electrostatic potential, $g=2 c_{s}^{2} / R$ is the effective gravity force, $R$ is the tokamak major radius, $A_{j \|}=-J_{\|} /(n e)-e A_{\|} /\left(m_{e} c\right)$ is the magnetic vector potential with an electron inertia term included, $\sigma_{\|}=n e^{2} \tau_{e i} /\left(0.51 m_{e}\right)$ is the plasma conductivity parallel to the magnetic field, $\tau_{e i}=3 m_{e}^{2} v_{e}^{3} /\left(4 \sqrt{2 \pi} n e^{4} \ln \Lambda\right)$ is the electron-ion collision time. The vector potential $A_{\|}$is calculated from the inversion of $\nabla_{\perp}^{2} A_{\|}-\left(\omega_{p e}^{2} / c^{2}\right) A_{\|}=(4 \pi e n / c) A_{j \|}$. Then the parallel current density can be calculated from the relation $J_{\|}=-n e A_{j \|}-n e^{2} A_{\|} /\left(m_{e} c\right)$.

The boundary conditions for the electrostatic potential at the boundaries of simulation domain can be taken to be either conventional sheath limited conditions or simple periodic conditions. Zero-gradient conditions were applied at the z-directed boundaries for the density equation.

The physical parameters of high beta plasma, where the Alfvén physics becomes important, should have large beta such that $\tilde{\beta}>1$, and large Alfvén time, $\tau_{A} / \tau_{\perp}>>1$. At the 
same time, a fluid approximation $\left(\lambda_{e} / L<<1\right.$, where $\lambda_{e}$ is the electron mean free path) also should be satisfied for our fluid model to be valid.

\section{Linear blob stability analysis}

To get an insight in the onset of plasma turbulence on a blob dynamics we perform a local linear stability analysis neglecting, for simplicity, the gravity (curvature) effects. Then, after some algebra, we find the following dispersion equation

$$
\Omega^{2}\left[\Omega-\chi+\Omega \widetilde{\beta}^{-1} \chi^{2}+i \Omega_{R} \chi^{2}\right]=\Omega_{A}^{2}\left[\Omega\left(1+\chi^{2}\right)-\chi\right],
$$

where $\Omega \equiv \omega / \hat{\omega}_{*}, \Omega_{R}=c^{2} /\left(4 \pi \sigma_{\|} \rho_{s}^{2} \hat{\omega}_{*}\right), \Omega_{A}=k_{\|} v_{A} / \hat{\omega}_{*}$ are normalized frequencies with a normalizing parameter $\hat{\omega}_{*} \equiv c_{s} / \delta$, and $\chi \cong k_{y} \rho_{s}$ is a normalized perpendicular wave number with $k_{x} \rho_{s}<<1$ approximation. We notice that in the brackets on the left-hand side of Eq. (4) two first terms correspond to the electromagnetic response with respect to $\partial A_{\|} / \partial t$, and $\left(\nabla A_{\|} / B_{0}\right) \times \hat{b}_{0} \cdot \nabla \ln n$ respectively, while two last terms are due to the electron inertia ( $\tilde{\beta}^{-1} \chi^{2} \Omega$, which, in particular, describes the "unfrozeness" of magnetic field on a scalelength of the order of collisionless skin depth) and plasma resistivity.

We start the analysis of Eq. (4) with the electrostatic limit as a reference. If the resistivity term, $i \Omega_{R} \chi^{2}$, is large enough so that $\Omega_{R} \sim \delta \cdot B^{2} / T_{e}^{3}>>1+\widetilde{\beta}^{-1}$, the electromagnetic response terms can be neglected and we come to a standard dispersion equation for electrostatic resistive drift waves without electron inertia:

$$
\Omega^{2} \Omega_{R} \chi^{2}+i \Omega_{A}^{2}\left[\Omega\left(1+\chi^{2}\right)-\chi\right]=0
$$

In this case, the maximum growth rate for the drift wave is found to be $\gamma_{\max } \sim 0.15 \hat{\omega}_{*}$ without a dependency on $\Omega_{R}$ for $\Omega_{A}^{2} / \Omega_{R} \cong 0.24$ with $\chi=1$. 
Next, we consider the electromagnetic case neglecting both plasma resistivity and electron inertia terms. For $\Omega_{A}^{2}>>1$ and $\chi \sim 1$, we obtain dispersed Alfvén waves $\Omega^{2}=\Omega_{A}^{2}\left(1+\chi^{2}\right)$ and a drift wave $\Omega=\chi /\left(1+\chi^{2}\right) \leq 1<<\Omega_{A}$. For $\Omega_{A}^{2}<<1$, the dispersion relation yields two Alfvén waves $\Omega^{2}=\Omega_{A}^{2}$ and a drift wave $\Omega=\chi$. Without the resistivity term and the electron inertia term all these waves are stable. However, a small resistive correction $\left(\Omega_{R}<1\right)$ makes the drift wave unstable. Neglecting the electron inertia term, we find that: for $\Omega_{A}^{2}>>1$, the growth rate of the resistive drift wave is estimated as $\gamma / \hat{\omega}_{*}(\chi \sim 1) \sim \Omega_{R} \chi^{4} /\left(\Omega_{A}^{2}\left(1+\chi^{2}\right)^{3}\right)$ and $\gamma / \hat{\omega}_{*}(\chi>>1) \sim \Omega_{R} /\left(\Omega_{A} \chi\right)^{2} ;$ for $\Omega_{A}^{2}<<1$, the growth rate is approximately $\gamma / \hat{\omega}_{*}(\chi>>1) \sim\left(\Omega_{A}^{2} \chi \Omega_{R} /\left(1+\Omega_{R}^{2} \chi^{2}\right)\right)^{1 / 2}$. Thus, in contrast to the electrostatic case where $\gamma_{\max } / \hat{\omega}_{*} \sim O(1)$ and is independent of $\Omega_{R}$, the electromagnetic case exhibits a strong reduction in the growth rate: $\gamma_{\max } / \hat{\omega}_{*} \tilde{<} \Omega_{R}<<1$. We verified the results of our analytic analyses by numerical solution of equation (4) (see Fig.1). This "stabilization" of drift wave instability due to electromagnetic effects is also observed in the BOUT $++[4]$ simulation of high-beta blob dynamics (see Fig.2).

The electron inertia effects become important if plasma beta is small, $\tilde{\beta}^{-1}>>1$ and the term $\tilde{\beta}^{-1} \chi^{2} \Omega$ in the first bracket dominates. Then, the maximum growth rate for this instability is $\gamma / \hat{\omega}_{*} \sim 0.3$ reached for $\Omega_{A}^{2} \tilde{\beta} \sim O(1)$ with $\chi \sim 1$. However, the $\tilde{\beta}^{-1} \chi^{2} \Omega$ dominated situation where low-beta and high temperature conditions are satisfied, the fluid approximation is not valid because $k_{\|} \lambda_{e}>1$. (See Fig. 3). Both electromagnetic and electron inertia effects become negligible roughly when $\Omega_{R} \widetilde{>} 1+\widetilde{\beta}^{-1}$ (See Fig. 4).

So far, we neglected the $E \times B$ convective motion due to the effective gravity force to focus on the electromagnetic effect on the drift wave instability. However, the variation of the spatial blob shape due to the macroscopic blob evolution also has a significant impact on the 
blob stability. The blob shape evolution depends on many ingredients (e.g., the sheath dissipation). The impact of resistive drift instability on blob dissipation is important only if the growth rate is larger than the critical time rate of the blob shape deformation, i.e. $\gamma \cdot \tau_{c r i t}>1$. For the case of no sheath dissipation effects on a blob macro-dynamics we have $\tau_{c r i t} \sim \sqrt{n_{b} \delta /\left(n_{a} g\right)}$ [5], where $n_{b}$ and $n_{a}$ are density of the blob and the ambient plasma respectively. As an example, this condition gives $\delta \cdot R>10^{3} \mathrm{~cm}^{2}$ for high beta plasma with $n_{b}=1 \times 10^{15} \mathrm{~cm}^{-3}, n_{a}=5 \times 10^{14} \mathrm{~cm}^{-3}$ and $T_{e}=200 \mathrm{eV}$. Therefore, the drift wave instability for typical size $(\sim 1 \mathrm{~cm})$ high beta blob is negligible in conventional tokamaks but might be marginally important for future tokamaks. This condition depends on dynamic variables such as electron and ion temperature. Therefore, the decrease of temperature due to the parallel heat conduction in SOL should be accounted for more precise estimation.

\section{Electromagnetic effects on macroscopic blob evolution}

In previous section, we described electromagnetic effects on microscopic blob stability not specifying the boundary conditions. However, physically, the parallel boundaries of blob filaments should be connected to something. Even though there is some uncertainty in what boundary condition is most appropriate for a high beta blob just after its formation, we first consider the widely used sheath-limited boundary condition. In the electrostatic approximation, the parallel current dipoles that drain the polarized charges to the sheath boundary, are almost immediately formed, with $J_{\|}(z)=2 n e^{2} c_{s} \phi z /\left(L_{\|} T_{e}\right)$ satisfying the sheath current limit. In the electromagnetic theory, however, the propagation speed of the current is limited by the Alfvén speed. Therefore, if the Alfvén time is larger than the blob propagation time, the parallel current dipoles are localized near the sheath region while the blob is being polarized continuously. Moreover, the higher conductivity, due to the higher temperature, will 
lengthen the magnetic diffusion time, and this will act as an effective inductance inhibiting the evolution of the magnetic field. Consequently, when $\tau_{A} / \tau_{\perp}>>1$, sheath connected high-beta blobs are effectively disconnected from the sheath and evolve similarly to small sheath limited blobs $\left(\delta<\delta_{*}\right)$ and form a mushroom-like shape.

The parallel inhomogeneity of the dipole potentials in high beta blobs may lead to bending of the blob filaments due to relatively long parallel Alfvén time. Both a longitudinal density bump in blob filament and a magnetic curvature variation can result in inhomogeneous electric and magnetic potentials along field line. The inhomogeneous polarization makes local deviations of convection speeds because the potential homogenizing time (Alfvén time) along blob filament is longer than the perpendicular blob propagation time. This bending of a blob filament is associated with field line bending since the magnetic field diffusion time is relatively long in high temperature plasma.

Figure 5 shows the density evolution obtained with the BOUT ++ simulations for a highbeta blob filament within an inhomogeneous curvature field and the magnetic field lines for different models: (a)electromagnetic model (Eqs. 1-3), and (b)electrostatic model (Eqs. 1-2 and Ohm's law). The magnetic perturbations are calculated from the inversion of the parallel current for the electrostatic model. In the case of the electromagnetic model, the magnetic field is strongly coupled, or frozen-in, to the plasma filament while the filament is freely crossing the magnetic field lines in the electrostatic calculation. Moreover, the plasma filament shows bending motion in the electromagnetic model while the electrostatic model shows flat homogeneous propagation of blob filament. This bending can cause the filament to touch the first wall directly while the ends could still stay in the relatively hot plasma region $[6]$.

\section{Conclusion}


We consider both microscopic and macroscopic impacts of electromagnetic effect on blob dynamics. We demonstrated with both linear stability analysis and nonlinear BOUT++ simulations that the resistive drift wave instability is suppressed by electromagnetic perturbations in high temperature or high beta plasmas. Only a small growth rate proportional to resistivity remains for resistive drift waves in high beta regime. As a result, drift wave turbulence cannot dissipate high-beta blobs. However, the dependency on resistivity disappears and drift-wave growth rate strongly increases when the plasma enters the electrostatic regime when resistivity exceeds some critical value. We note that the blob motion into the SOL is accompanied by reduction of the electron temperature and enhancement of the resistive effects, so the blob can switch from the electromagnetic to the electrostatic regimes.

The second major finding is that the inhomogeneity of the magnetic curvature or the blob pressure along the blob filament leads to bending of the filaments, in the high-beta case. This is because the propagation time of the electric current (the Alfvén time) increases in higherdensity plasma. The effects of sheath boundary conditions on the part of the blob away from the boundary are also diminished by the increased Alfvén time.

\section{Acknowledgements}

This material is based upon work supported by the U.S. Department of Energy Office of Science, Office of Fusion Energy Science under Award Number DE-FG02-04ER54739 and DE-SC0010413 at UCSD. This research was also supported by the Kwanjeong Educational Foundation. 


\section{References}

[1] S. Krasheninnikov, D. D’Ippolito, J. Myra, J. Plasma Phys. 74 (2008) 679.

[2] D. D’Ippolito, J. Myra, S. Zweben, Phys. Plasmas 18 (2011) 060501.

[3] J. Angus, S. Krasheninnikov, M. Umansky, Phys. Plasmas 19 (2012) 082312.

[4] M. Umansky, X. Xu, B. Dudson, L. LoDestro, J. Myra, Comput. Phys. Commun. 180 (2009) 887.

[5] J. Angus, S. Krasheninnikov, "Evolution of large amplitude, localized density stratifications in an ambient background and a uniform gravity field", to be submitted for publication.

[6] S. Krasheninnikov, D. Ryutov, G. J. Yu, Plasma Fusion Res. 6 (2004) 139. 


\section{Figure captions}

Fig. 1. Normalized growth rate $\gamma / \hat{\omega}_{*}$ as function of $\Omega_{A}$ and $\chi$ for two different models: electromagnetic (EM) and electrostatic (ES) calculation are shown. Note that the EM growth rate is multiplied by 50 for clarity. The parameters $\Omega_{R}$ and $\tilde{\beta}^{-1}$ correspond to a high beta plasmas, $n_{0}=5 \times 10^{14} \mathrm{~cm}^{-3}, T_{e}=200 \mathrm{eV}$.

Fig. 2. Blob density contours of high-beta blob plasma $\left(n_{0}=5 \times 10^{14} \mathrm{~cm}^{-3}, T_{e}=200 \mathrm{eV}\right)$ from the BOUT++ simulations for EM (top) and ES (bottom) models. The electrostatic model with Ohms' law shows the resistive drift wave. The effective gravity force is suppressed for simplicity.

Fig. 3. Normalized maximum growth rate, $\max \left(\gamma / \hat{\omega}_{*}\right)$, contours as function of $\Omega_{R}\left(\propto T_{e}^{-3}\right)$ and $\widetilde{\beta}^{-1}\left(\propto n^{-1} T_{e}^{-1}\right)$ are calculated using Eq. (4). The maximum growth rate is decreased significantly as temperature and density increase. The dotted line represents $k_{\|} \lambda_{e}=1$ with respect to $\Omega_{A}$ where the maximum growth rate was measured.

Fig. 4. Maximum normalized growth rate $\gamma / \hat{\omega}_{*}$ as a function of resistivity parameter $\Omega_{R}$ for electromagnetic blobs of three different values of $\widetilde{\beta}^{-1}=0.1,1.0,10$ and electrostatic blob with electron inertial neglected are shown. Electromagnetically modified instabilities return to the electrostatic resistive drift wave instability as resistivity increases. 
Fig. 5. Comparison of the BOUT ++ simulation of high-beta blob evolution in time sequences within an inhomogeneous curvature field for (a)EM model and (b)ES model are shown. The 2D density contours are taken at five different positions along z-direction (not in real scale). The solid lines (blue, red) along z-direction represent the magnetic field lines. The red solid line represents a magnetic field line which was threading the blob filament at $t=0$. 


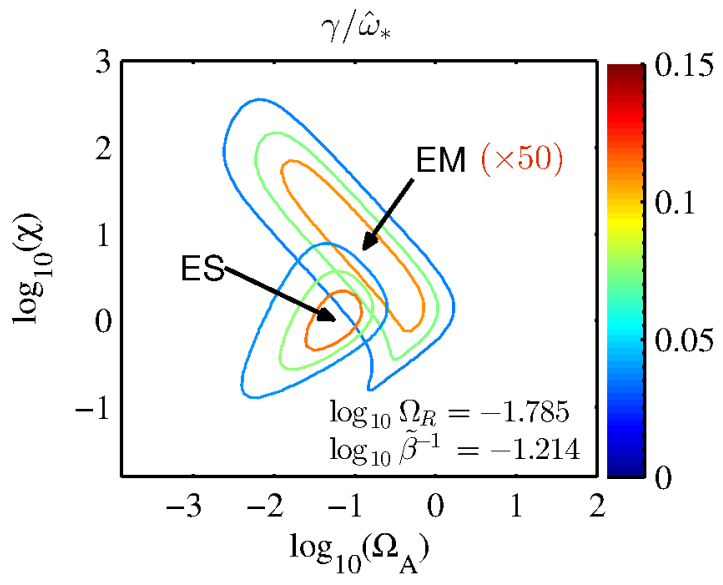

Fig. 1. 


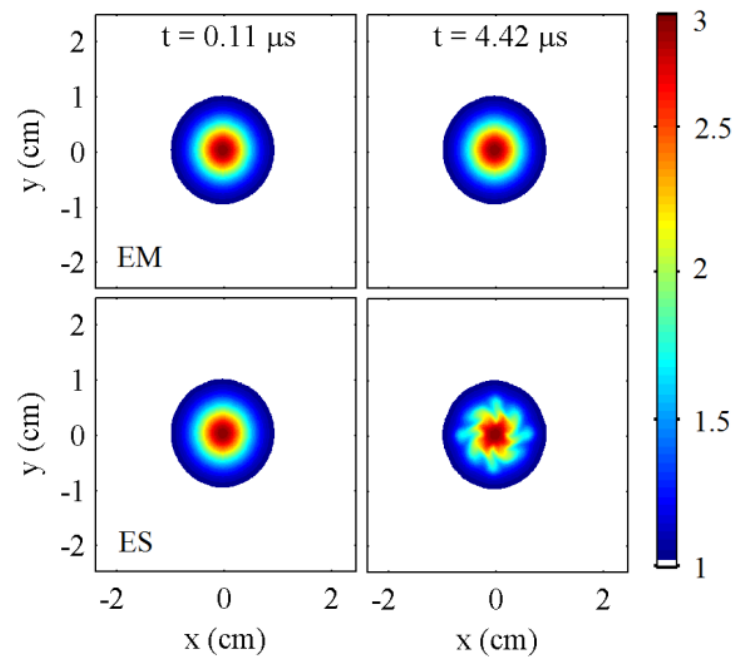

Fig. 2. 


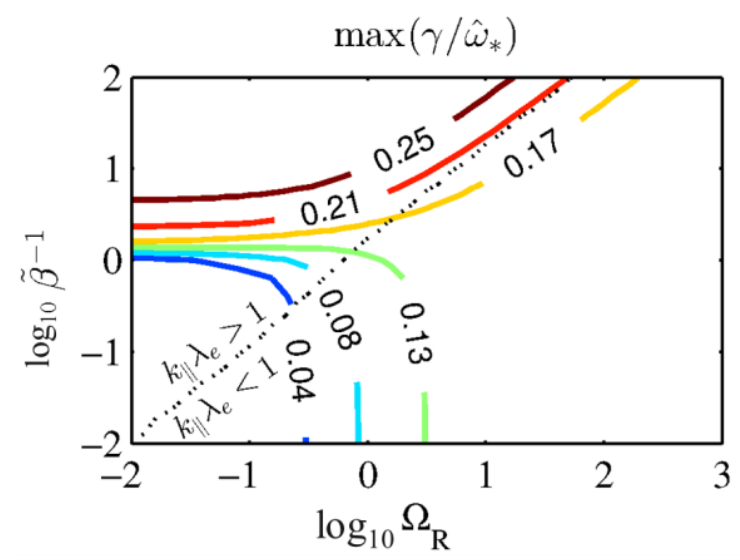

Fig. 3. 


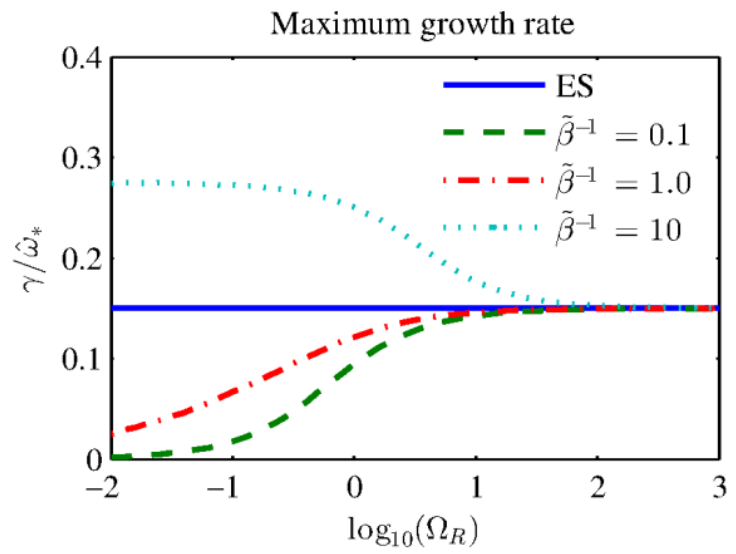

Fig. 4. 
(a)

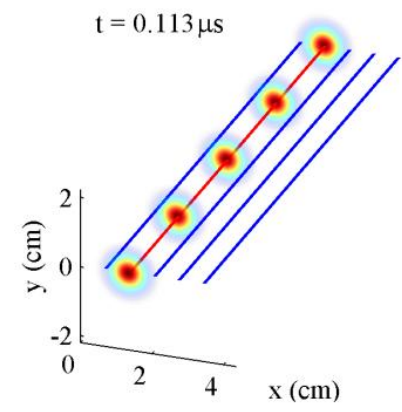

(b)

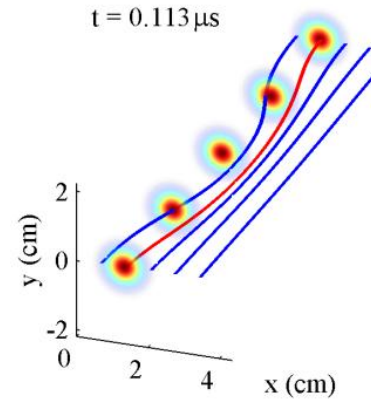

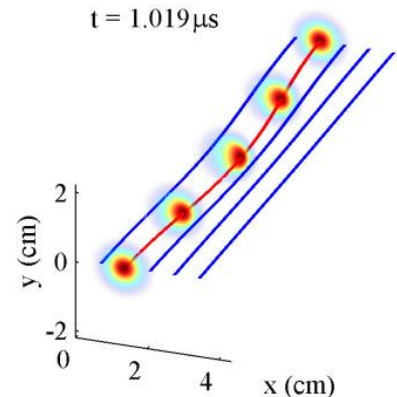

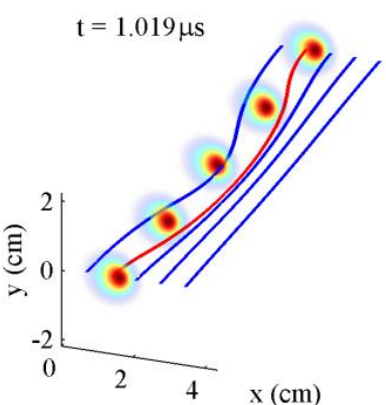

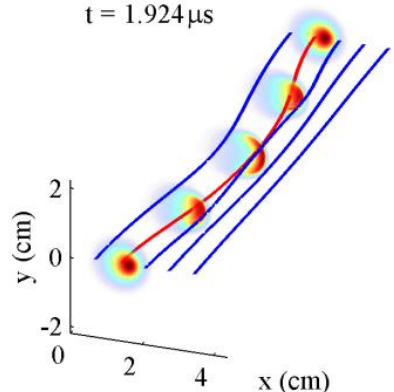

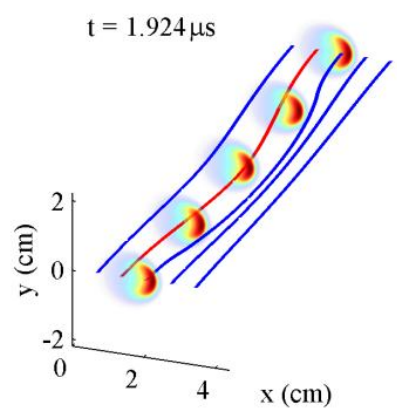

Fig. 5. 